\title{
Light Controlling Light in an Optical Fibre: From Very Slow to Faster-Than-Light Speed
}

\author{
Luc Thévenaz, Kwang-Yong Song, Sang-Hoon Chin and Miguel Gonzalez-Herraez \\ Laboratoire de Nanophotonique et Métrologie, Ecole Polytechnique Fédérale de Lausanne (EPFL), \\ STI-NAM Station 11, 1015 Lausanne, Switzerland
}

\begin{abstract}
We demonstrate a method to achieve an extremely wide and flexible external control of the group velocity of signals as they propagate along an optical fibre. This control is achieved by means of the gain and loss mechanisms of stimulated Brillouin scattering in the fibre itself. Our experiments show that group velocities below $71,000 \mathrm{~km} / \mathrm{s}$ on one hand, well exceeding the speed of light in vacuum on the other hand and even negative group velocities can readily be obtained with a simple benchtop experimental setup. Advanced schemes can be realized thanks to the extremely flexible possibility to shape the gain spectrum to make it optimized for applications. Ultra wide bandwidth, delaying with flat amplitude response and lower distortion were successfully demonstrated this way.
\end{abstract}

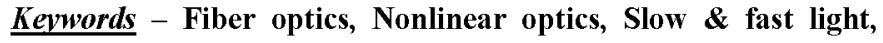
stimulated Brillouin scattering.

\section{INTRODUCTION}

The active control of the speed of a light signal in an optical fibre is attracting much attention for the development of fast-access memories and optically-controlled delay lines compatible with optical computing and fibre-optic communication systems. Recently a large effort has been carried out for realizing such an optically-controlled delay line in optical fibres, since they are believed necessary for the development of the future all-optical packet routers. Successful experiments to widely control the light group velocity have been reported these past few year [1], showing the possibility to slow the speed of light up to nearly stopping it or to achieve group velocity exceeding the vacuum light velocity $c[2,3]$. Strong negative group velocities have also been demonstrated [4]. But all these experiments use special media like cold atomic gases [5-7] or electronic transitions in crystalline solids ${ }^{[8]}$ working at well defined wavelengths. Previous works have demonstrated the possibility of achieving superluminal group velocities in optical fibres [9], but with no optical control. Here we summarize our work this past year demonstrating for the first time a wide optical control of the signal velocity in an optical fibre, realized with the recently suggested approach of using the narrow band gain or loss generated by a nonlinear optical interaction, the stimulated Brillouin scattering [10]. The high flexibility of this interaction makes this active control possible in any type of fibre and at any wavelength, in particular in the low loss window of optical fibres.

\section{THEORETICAL BACKGROUND}

Stimulated Brillouin Scattering (SBS) is usually described as the interaction of two counter propagating waves, a strong pump wave and a weak probe wave [11]. If particular phase matching conditions are met (namely $f_{\text {Pump }}=f_{\text {probe }}+v_{B}, v_{B}$ being the Brillouin shift), an acoustic wave is generated. This acoustic wave scatters photons from the pump to the probe wave, stimulating the process. From a practical point of view, the process of SBS can be viewed as a narrowband amplification process, in which a continuous-wave pump produces a narrowband $(30-50 \mathrm{MHz})$ gain in a spectral region around $f_{P_{u m p}} v_{B}$.

Assuming $f_{\text {Pump }}=f_{\text {probe }}+v_{B}$ and no pump depletion, the spatial evolution of the electric field amplitudes of pump $\left(A_{p}\right)$ and probe $\left(A_{s}\right)$ waves under SBS is described by the following coupled equations:

$$
\begin{aligned}
& \frac{d A_{p}}{d z}=-\frac{g_{B}}{2 A_{e f f}} \frac{\left|A_{s}\right|^{2}}{1-2 j\left(\frac{\Delta v}{\Delta v_{B}}\right)} A_{p}-\frac{\alpha}{2} A_{p} \\
& \frac{d A_{s}}{d z}=\frac{g_{B}}{2 A_{e f f}} \frac{\left|A_{p}\right|^{2}}{1+2 j\left(\frac{\Delta v}{\Delta v_{B}}\right)} A_{s}+\frac{\alpha}{2} A_{s}
\end{aligned}
$$

where $g_{B}$ is the Brillouin gain coefficient, $A_{\text {eff }}$ the mode effective area, $\Delta v$ the frequency deviation from $v_{B}, \Delta v_{B}$ the gain bandwidth and $\alpha$ the linear attenuation coefficient. The usual treatment of these equations in the literature has basically concentrated on the intensity increase of the probe 
wavelength, which can be described with the exponential law $I_{S}(L)=I_{s}(0) \exp \left(g_{B} I_{P} L\right)$. However, while the real parts of the equations are related with gain in the probe wave or loss in the pump wave, the imaginary parts are responsible for additional phase shifts undergone by the two waves [11]. More specifically, through the SBS process the pump wave induces a propagation constant change in the probe wave given by:

$$
\Delta \beta=\operatorname{Im}\left\{\frac{g_{B}}{2} \frac{I_{p}}{1+2 j\left(\frac{\Delta v}{\Delta v_{B}}\right)}\right\}
$$

where $I_{P}$ is the pump power intensity in the fibre. This change in the propagation constant has strong frequency dependence, as shown in Fig. 1. If we consider a pulse propagating at the probe wavelength, its velocity will be related to the group velocity $v_{g}=(d \beta / d \omega)^{-1}$. Thus, a sudden change in the propagation constant with frequency produces a strong change in the group velocity, which in turn introduces an additional delay of the pulse at the fibre output, $\Delta t=L / v_{g}$. If the frequency difference between pump and probe exactly matches the Brillouin shift $(\Delta v=0)$, the resultant optical time delay is given by $\Delta t=g_{B} I_{P} L /\left(2 \pi \Delta v_{B}\right)$. We can thus say that the delay varies logarithmically with the net gain (loss) experienced by the probe. A fast evaluation of these quantities in conventional single-mode fibres $\left(\Delta v_{B} \approx 35 \mathrm{MHz}\right)$ leads to a surprisingly simple rule-of-thumb: 1 ns delay is introduced per $\mathrm{dB}$ gain introduced in the probe.

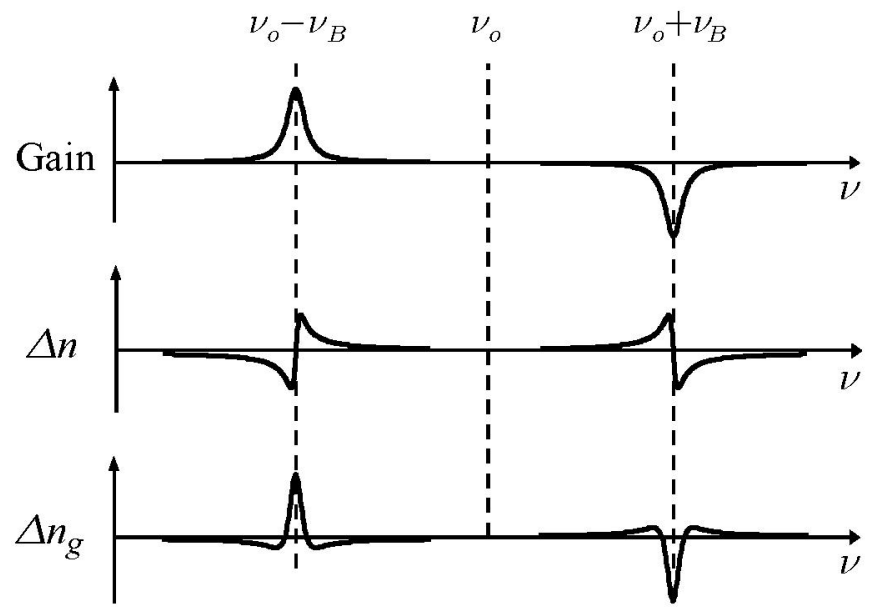

Fig. 1 Principle of signal delaying and advancement using stimulated Brillouin scattering.

So far we have treated the case of gain in the probe, this is $f_{\text {Pump }}=f_{\text {probe }}+v_{B}$. In this case, an extra delay is introduced in the probe signal with respect to the normal propagation time along the fibre. By tuning the probe wavelength so that $f_{\text {Pump }}=f_{\text {probe }}-v_{B}$ we observe loss at the probe wavelength. The treatment of the loss case is exactly the same to the gain case but changing the roles of pump and probe in the coupled equations, so the delay in this case is not positive but negative. This is viewed as an advancement of the pulse with respect to the conventional propagation along the fibre.

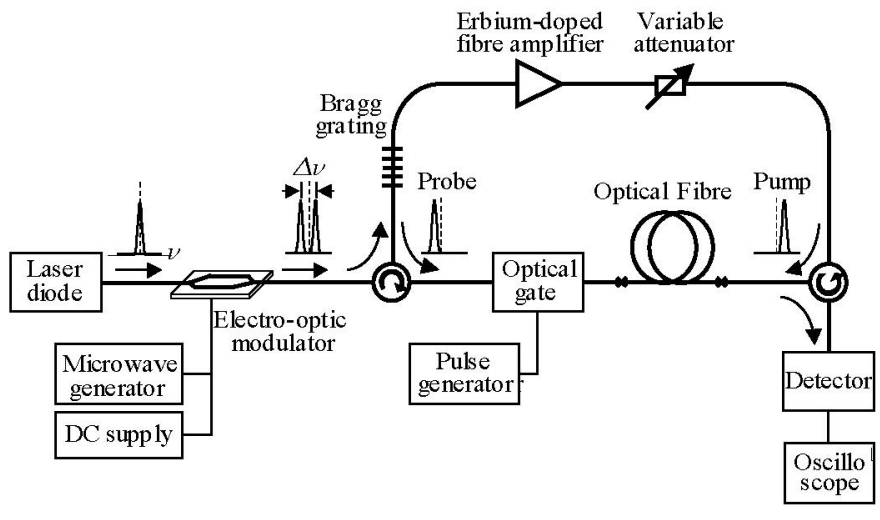

Fig. 2. Experimental configuration.

\section{EXPERIMENT AND RESULTS}

Figure 2 shows the experimental setup, in which the pump and probe signals are generated through the modulation of the light from one laser [12]. This results in an ideal stability as far as the frequency difference between pump and signal is concerned, that is essential regarding the narrow spectral width of the Brillouin gain. To properly observe the delay, a pulse probe signal is generated while the pump is a continuous wave $(\mathrm{CW})$. A DFB laser diode operating at $1552 \mathrm{~nm}$ was used as a light source and its output was launched into an electro-optic modulator (EOM) to create two first-order sidebands. The carrier wave was suppressed by controlling the DC bias voltage delivered into the EOM and the frequency difference between the two sidebands was set exactly to the Brillouin frequency $v_{B}$ of the test fibre, that is around $10.8 \mathrm{GHz}$ at this wavelength for standard fibres.

In order to measure the effect of Brillouin gain, the lowerfrequency sideband was reflected by a narrow band fibre Bragg grating and optically gated to be used as a probe pulse. For this purpose another EOM was used as a fast optical gate, resulting in clean pulses with sharp rising and trailing edges. The higher-frequency sideband was used as the $\mathrm{CW}$ Brillouin pump after being amplitude-controlled by a broadband erbium-doped fibre amplifier and a variable attenuator. The time delay of the probe pulse was measured for different Brillouin gains by varying the pump amplitude from zero to several tens of $\mathrm{mW}$. In the case of the Brillouin loss experiment, we swapped the roles of the two sidebands, so that the higher-frequency sideband was used to build the probe pulse and the lower frequency sideband was used to build the $\mathrm{CW}$ pump wave. Then, the measurement was performed in the same way by varying the power of the $\mathrm{CW}$ pump wave and monitoring the amplitude and the time delay of the probe pulse.

We first observed the delaying effect along a $11.8 \mathrm{~km}$ standard single mode fibre with the gain swept continuously from $0 \mathrm{~dB}$ to $30 \mathrm{~dB}$. The probe pulse width (FWHM) was $100 \mathrm{~ns}$ and the maximum pump power was about $40 \mathrm{~mW}$, limited basically by the threshold for amplified spontaneous Brillouin emission. We could see clear retardation of the 


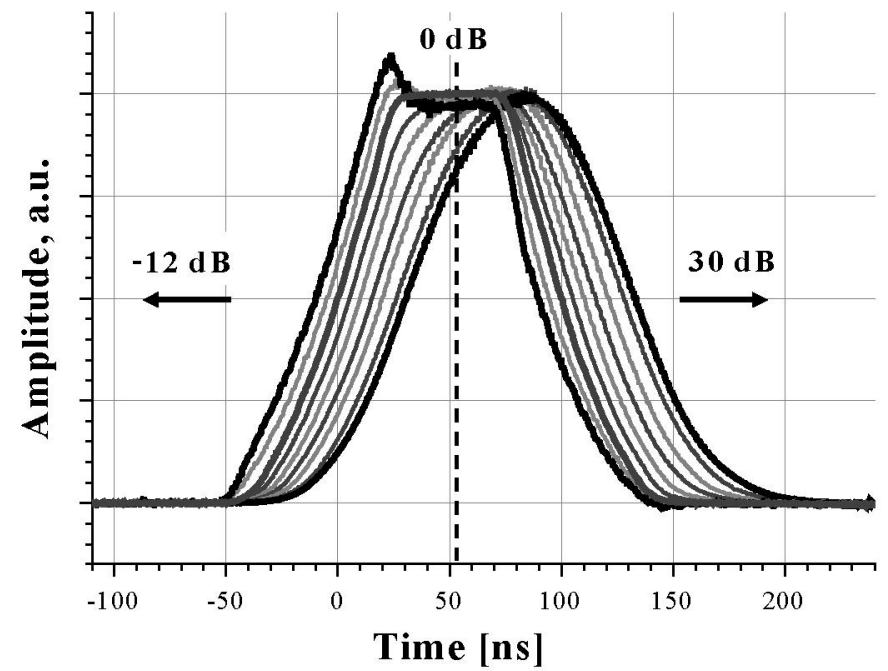

Fig. 3 Pulse waveforms and delay according to Brillouin gain and loss in $11.8 \mathrm{~km}$ standard fibre using $100 \mathrm{~ns}$ pulse.

pulse as the Brillouin gain increased and the maximum delay time was close to $30 \mathrm{~ns}$ when the gain was $30 \mathrm{~dB}$. This corresponds to a $7.6 \times 10^{-4}$ group index change and the delay varies logarithmically with the net gain with a slope of $1.07 \mathrm{~ns} / \mathrm{dB}$, in good agreement with the theoretical prediction. Pulse advancement was also clearly observed in the loss configuration with a maximum value of $-8.4 \mathrm{~ns}$ for a corresponding loss of $-12 \mathrm{~dB}$. A larger loss was practically difficult to obtain, non-attenuated residual signals at different wavelengths superposing with the probe pulse with comparable amplitudes. The time waveforms and the optical delay as a function of $\mathrm{dB}$-gain are shown in Fig. 3. The distortion of the pulse shape came from the filtering effect due to the narrow bandwidth.

Since the delay (advancement) only depends on the overall gain (loss) experienced along the full fibre length for a given type of fibre, the group index change can be drastically increased by realizing the same gain (loss) over a shorter fibre using a higher pump power. In other words, the index change will scale in the inverse proportion of the fibre length for a fixed gain (loss) to maintain the same delay. Actually the group index change will vary from the $10^{-3}$ range for kilometre-long fibres to the unity range for meter-long fibres. In this latter case it is thus possible to conceive a system with a group index smaller than 1 , hence faster than the vacuum light velocity, or even negative, with the pulse maximum emerging out of the fibre before it actually enters the fibre.

We could experimentally verify that this extreme situation can be actually realized [13]. The same experiment was carried out in a sample of $2 \mathrm{~m}$ of standard single mode fibre. The main issue was to raise the pump power to 10 Watt range to obtain a $30 \mathrm{~dB}$ gain using stimulated Brillouin scattering over the short fibre. This could be achieved by forming a pulse train with the pump wave, so that the full gain is available in the erbium-doped fibre amplifier while the average output power is kept below the saturation power. Thus an additional electro-optic modulator was used to gate the pump pulse. This gating was synchronized with the probe pulse and the pump pulse was made longer than twice the propagation time in the fibre sample, so that the signal pulse sees a constant pump power while propagating throughout the entire fibre sample.

Fig. 4-(a) shows time waveforms of pulses experiencing different gains and losses through stimulated Brillouin scattering in the short fibre. The observed delays in this $2 \mathrm{~m}$ sample are fully comparable to those obtained along several kilometres of fibre. Fig. 4-(b) shows the pulse peak position as a function of the gain (loss) experienced by the signal and the equivalent group index change. As it can be seen, the group index could be increased continuously from 1.46 in normal conditions to 4.26 under high Brillouin gain, and lowered to -0.7 under high Brillouin loss, a negative group index meaning that the pulse peak exits the fibre before it enters. In other words, the group velocity could be changed continuously from $70^{\prime} 500 \mathrm{~km} / \mathrm{s}$ to infinite and then to $428^{\prime} 000 \mathrm{~km} / \mathrm{s}\left(\sim 205^{\prime} 000 \mathrm{~km} / \mathrm{s}\right.$ in normal conditions), leading to impressive delays from $-14.4 \mathrm{~ns}$ to $+18.6 \mathrm{~ns}$ in only 2 meter of fibre. In terms of length, this means that the fibre effective length can be continuously changed from $-3 \mathrm{~m}$ to $3.8 \mathrm{~m}$.

It must be pointed out that a group velocity faster than the
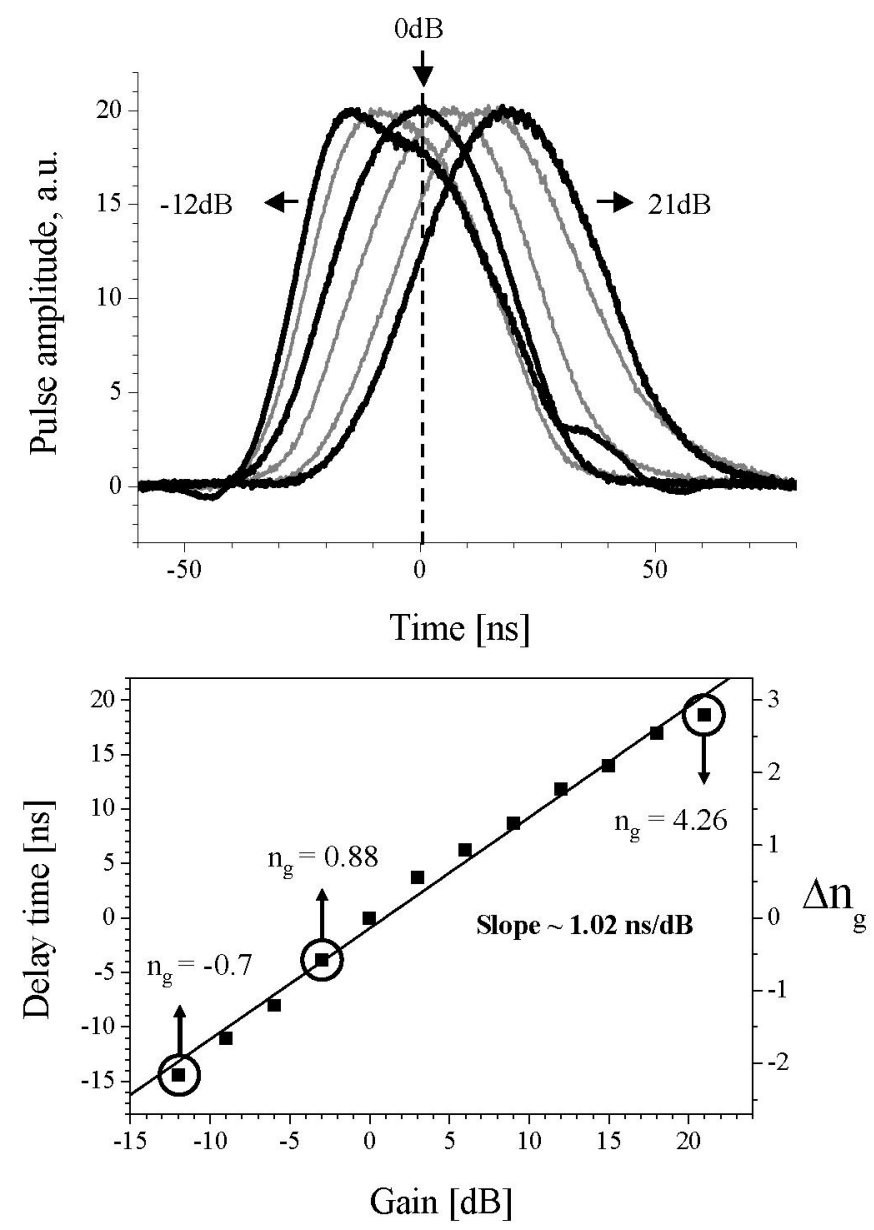

Fig. 4. Very large group velocity change achieved in a $2-\mathrm{m}$ standard fibre using $40 \mathrm{~ns}$ pulse.

Top: pulse waveform at the fibre output.

Bottom: pulse delay and group index modification; the absolute group index is shown for extreme velocities (slowest, superluminal \& negative) 
vacuum light velocity $c$ or even negative does not break the famous principles resulting from causality and relativity. The fact that the spectral transition is narrowband prevents all frequency components from experiencing the same group velocity and amplitude response, so that the information cannot finally propagate at a speed faster than $c$ [5]. This leads to a severe distortion of the pulse in the case of fast light (starting and ending points at normal light velocity in the medium and non-attenuated, peak point at modified group velocity and attenuated), resulting in a steeper leading edge and a longer trailing edge. This can be clearly observed in Fig. 4-(a) in the case of strong pulse advancement. In the case of pulse delaying, the peak of the pulse is substantially amplified with respect to the leading and trailing edges, and the symmetry of the pulse appears better preserved.

In order to extend the time delay, we introduced a new configuration composed of cascaded fibre segments including unidirectional optical attenuators in the fibre junctions as shown in Fig. 5 [14]. Four uniform fibre spools with the same Brillouin frequency and length of $1.1 \mathrm{~km}$ were used as gain media, and they were cascaded using unidirectional variable attenuators as shown in the inset.

In this configuration, the amplification of the probe pulse was periodically compensated by the variable optical attenuator (VOA), while the counter-propagating pump wave did not experience the attenuation. This feature helped avoiding the gain saturation coming from large amplification of the pulse, maintaining the induced optical delay. In addition, the depletion of the strong pump wave due to ASBE was also avoided by the periodic step absorption of the $\mathrm{CW}$ backscattered wave.

The time waveforms of the probe pulses are shown in Fig. 6 for a gain swept from $0 \mathrm{~dB}$ to $120 \mathrm{~dB}$. A Gaussianshaped pulse was used as the probe with an initial pulse width (FWHM) of $42 \mathrm{~ns}$. Clear time delay was observed as the gain increased and the maximum delay of $152 \mathrm{~ns}$ was achieved when the gain was $120 \mathrm{~dB}$, which corresponds to 3.6 times the initial pulse width. In the mean time, a considerable broadening of the pulse from $42 \mathrm{~ns}$ to $102 \mathrm{~ns}$ was also observed which is attributed to the narrow gain.

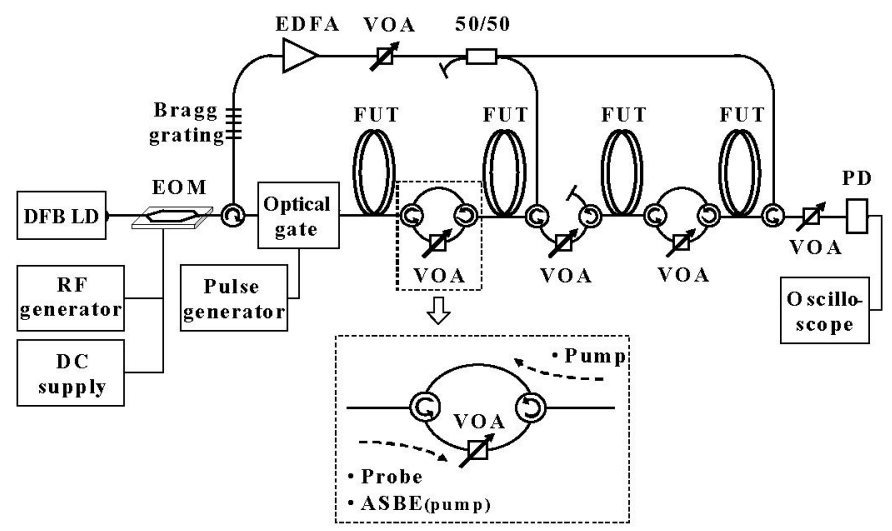

Fig. 5 Experimental setup using cascaded fibre structure and unidirectional attenuators.

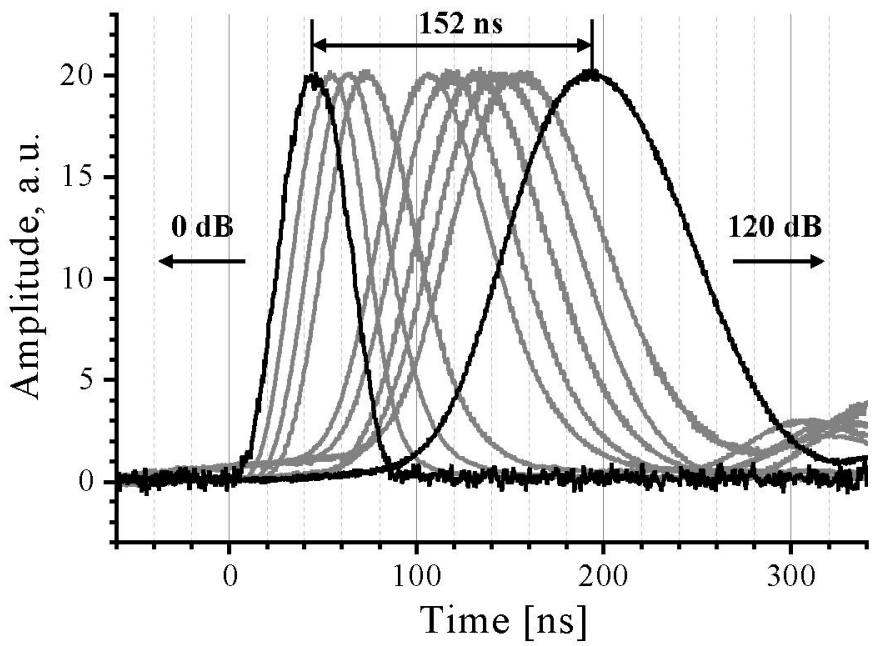

Fig. 6 Time waveforms of the probe pulses for different Brillouin gains, showing a time delay much exceeding the initial pulse width (42 ns)

\section{ADVANCED SCHEMES USING SPECTRAL SHAPING}

More recently stimulated Brillouin scattering (SBS) has proved to be an unprecedented and unmatched flexible tool for the generation of slow light regarding its spectral tailoring capability. Indeed, a large variety of gain spectral profile can be obtained by properly modulating the pump spectrum. When a monochromatic pump is used in the stimulated Brillouin interaction, the gain window appearing in the fiber transmission spectrum shows a Lorentzian shape whose characteristic spectral FWHM width is around $30 \mathrm{MHz}$ in conventional single-mode fibers pumped at $1.55 \mu \mathrm{m}$. However, when the pump is modulated the gain bandwidth is given by the convolution of the pump spectrum and the Brillouin gain curve. Hence the effective Brillouin gain spectrum $g(\Delta v)$ is given by:

$$
g(\Delta v)=P(\Delta v) \otimes g_{B}(\Delta v)
$$

where $\otimes$ denotes convolution, $P(\Delta v)$ is the normalized pump power spectral density (i.e. its integral is unity) and $g_{B}(\Delta v)$ is the natural Lorentzian gain of the Brillouin amplification process.

The effect of this convolution is shown in Fig. 7. By properly modulating the pump signal, it is possible to generate nearly any gain spectral distribution and to optimize the slow \& fast light effect in terms of bandwidth, efficiency and distortion.
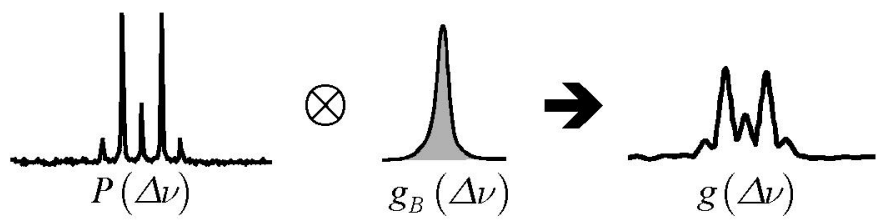

Fig. 7 The effective gain spectrum $g(\Delta v)$ is given by the convolution of the pump spectrum $P(\Delta v)$ with the natural gain distribution $g_{B}(\Delta v)$ of stimulated Brillouin scattering.

This offers the possibility to synthesize nearly any gain spectral distribution. 


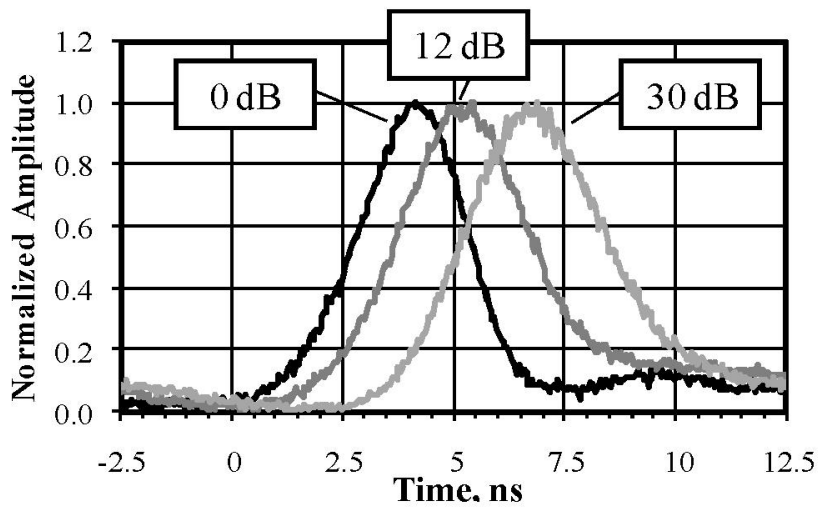

Fig. 8 Pulse delaying obtained using a $10 \mathrm{X}$ broadened gain spectrum The pulse width is $2.7 \mathrm{~ns}$.

An essential step towards applications was made when it was demonstrated that the bandwidth of SBS-based slow light can be made arbitrarily large by actively broadening the pump spectrum using random direct current modulation of the pump laser [15]. A particularly useful case arises when the pump spectrum can also be approximated by a Lorentzian distribution. In such conditions, the effective Brillouin gain shape remains Lorentzian, but shows a width equal to the sum of the characteristic Brillouin gain width and the pump spectral width. Hence an adequate pump modulation can be used to broaden at will the Brillouin interaction, suppressing the inherent limitation related to the narrow natural linewidth.

Fig.8 shows the delaying results of 2.7-ns pulses for several gain values. A linear dependence of the delay with the logarithmic gain is observed with a slope of approximately $0.092 \mathrm{~ns} / \mathrm{dB}$, as expected from the theory. By tuning the probe frequency appropriately, measurements in the Brillouin loss region were also performed, obtaining pulse advancement with the same linear dependence on the logarithmic gain.

This scheme was then replicated and improved successfully to recently ultimately reach a $10 \mathrm{GHz}$ bandwidth [16]. It was also demonstrated that the slowing efficiency can be substantially improved by departing from the Lorentzian shape to a more efficient finely tuned gain spectral distribution [17].

So far all these slow-light techniques suffer from the drawback of a significant amplitude change associated with the delaying effect. For instance a one-pulse width delaying gives rise to a large $30 \mathrm{~dB}$ pulse amplitude change using SBS. We have recently demonstrated that the high flexibility of SBS opens unprecedented achievements and offers the possibility to synthesize a gain spectral profile, so that signal delays or advances can be realized with an ideally absolute null amplitude change [18]. This can be obtained by the combination of gain and loss spectral profiles with identical depth but different width, resulting in a net zero gain and a differential delaying effect.

This is practically achieved by superposing in the frequency domain a SBS gain with linear gain $+G_{1}$ and a bandwidth $\Delta v_{1}$ on a SBS loss with negative linear gain (thus loss) $-G_{2}$ and a bandwidth $\Delta v_{2}$. They are generated using 2 distinct pump lasers placed at a frequency $\pm v_{B}$ above and

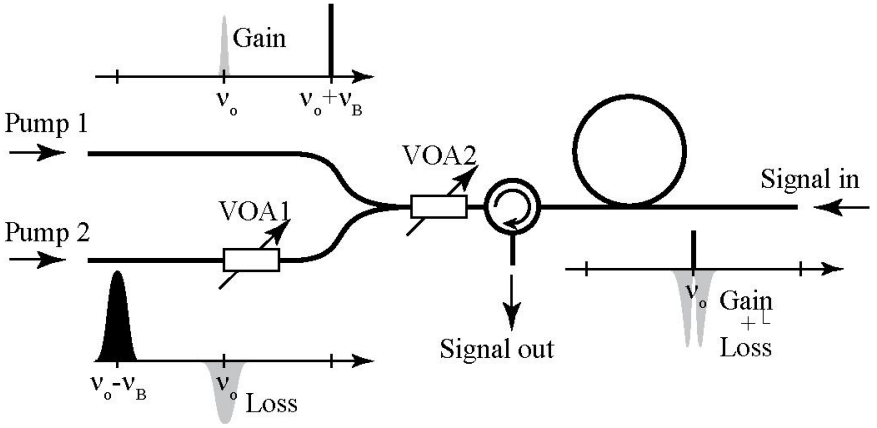

Fig.9. Principle of the experimental configuration to generate zero-gain spectral resonances where two distinct optical pumps were used to produce Brillouin gain and loss, respectively.

below the signal frequency $v$, respectively, as shown in Fig. 9. The bandwidth of each pump $\Delta v$ can be arbitrarily broadened and controlled using the scheme described above [15].

If the bandwidths of the gain and loss spectra are substantially different, e.g. $\Delta v_{2} » \Delta v_{1}$, it is possible to obtain a significant time delay with nevertheless a zero linear gain. The effect is fully comparable to electromagnetically-induced transparency or coherent population oscillation, in which a transparency window is opened in the middle of an absorption line. Fig. 10 shows the obtained gain/loss spectral profile, demonstrating that a good compensation of gain and loss can actually be obtained. A situation identical to ideal electromagnetically-induced transparency (EIT) can be achieved, i.e. up to full transparency.

Delays and amplitudes were recorded for different pump levels and are shown in Fig. 11. Delays comparable to the standard method [11] are obtained in a $2 \mathrm{~km}$ fiber, together with a maximum amplitude change of $\pm 1 \mathrm{~dB}$ that can be reasonably considered as a flat response. An equivalent delay using the non-compensated standard technique would result in a $12 \mathrm{~dB}$ amplitude change.

The gain compensation technique can also be applied to generate fast light, by simply swapping the frequency positions of Pump 1 and Pump 2. In this case the broadened

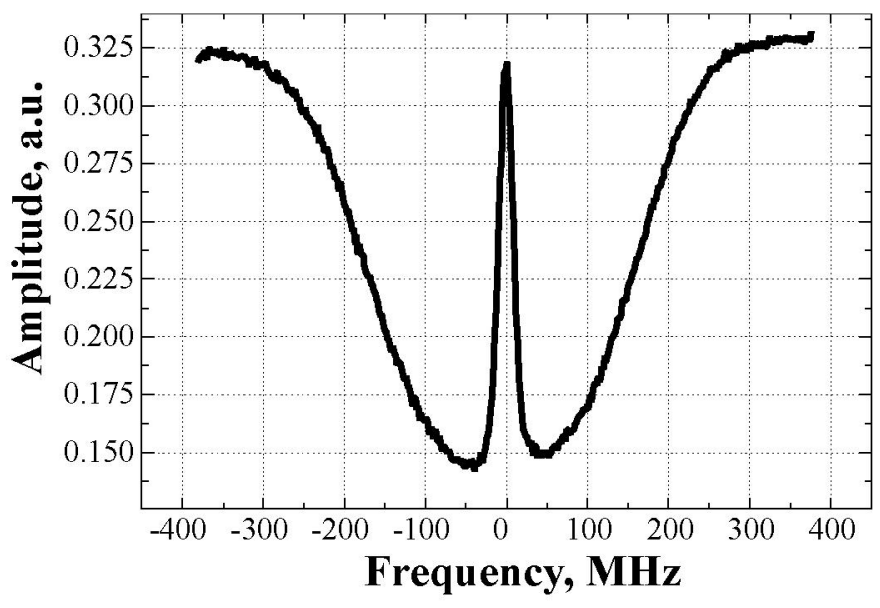

Fig.10 Variation of the amplitude of the probe signal as a function of frequency after propagation through a $2 \mathrm{~km}$ fiber, showing the achievement of a well-compensated SBS gain/loss profile. 


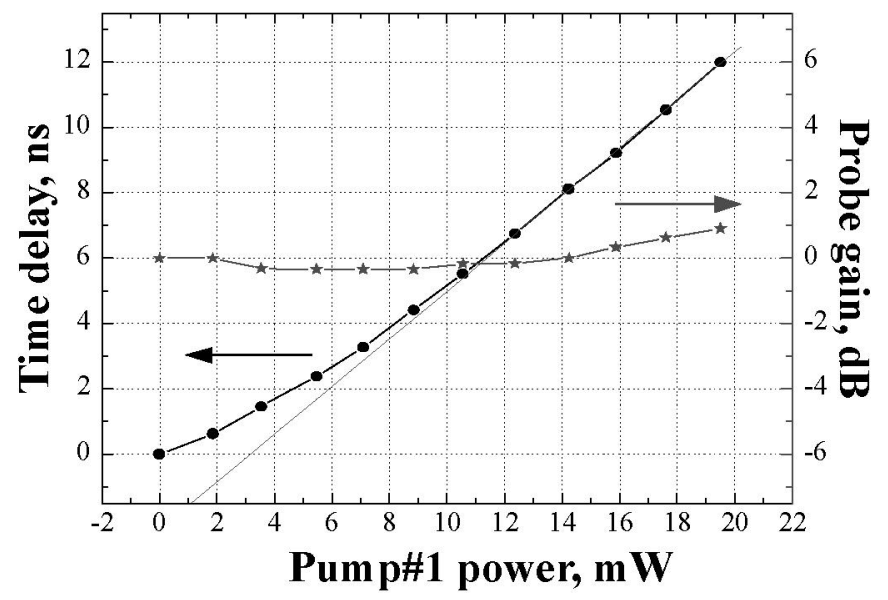

Fig. 11. Delays and amplitudes for a $1 \mathrm{MHz}$ sine modulated signal as a function of Pump 1 power in a zero-gain slow light configuration. Power of Pump 2 is 12 times larger than power of Pump 1.

pump generates a gain and the narrow pump burns a hole at the center of the gain spectrum. This configuration was also successfully experimentally tested and demonstrated [18].

The smart combination of gain and loss spectra using a multiple pump scheme was also used to extend the bandwidth well beyond $10 \mathrm{GHz}$ that was temporarily considered like the ultimate limit [19]. A $25 \mathrm{GHz}$ bandwidth was experimentally demonstrated that can be potentially extended well beyond using additional pumps.

\section{CONCLUSIONS}

A wide control of the group velocity of light signals in optical fibres has been demonstrated using stimulated Brillouin scattering. With this effect we have achieved nearly all results obtained using atomic transitions, from delays widely exceeding the optical pulse duration to superluminal propagation and even negative group velocity. These experiments can be realized on a tabletop in normal environmental conditions.

Stimulated Brillouin scattering also shows unprecedented advantages for the generation of slow \& fast light regarding its spectral tailoring capabilities. Through a smart combination of modulation and multiple pump gains supersposition it was demonstrated that delays with no amplitude change, enhanced slow light effect and ultra-wide bandwidth can be realized. This is a significant step towards making 'slow \& fast' light not only a scientific curiosity, but a real engineering tool.

\section{ACKNOWLEDGMENT}

This research was supported by several Institutions: the Swiss National Science Foundation through project 200021109773, the Post-doctoral Fellowship Program of Korea Science \& Engineering Foundation (KOSEF), the Spanish Ministry of Education and Science through grant TIC200301869 and the University of Alcalá through project UAH PI2005/076

\section{REFERENCES}

[1] R. W. Boyd and D. J. Gauthier, in Progress in Optics, edited by E. Wolf, Vol. 43, Chap. 6, p.497.

[2] V. Hau, S. E. Harris, Z. Dutton, and C. H. Behroozi, Nature (London) 397, 594 (1999).

[3] C. Liu, Z. Dutton, C. H. Behroozi, and L. V. Hau, Nature (London) 409, 490 (2001).

[4] L. J. Wang, A. Kuzmich, and A. Dogariu, Nature (London) 406, 277 (2000).

[5] M. D. Stenner, D. J. Gauthier, and M. A. Neifeld, Nature (London) 425, 695 (2003).

[6] A. M. Akulshin, S. Barreiro, and A. Lezema, Phys. Rev. Lett. 83, 4277 (1999).

[7] M. S. Bigelow, N. N. Lepeshkin, and R. W. Boyd, "Superluminal and slow-light propagation in a roomtemperature solid," Science 301, 200-202 (2003).

[8] N. Brunner, V. Scarani, M. Wegmuller, M. Legre, N. Gisin, Phys. Rev. Lett. 93, 203902-1 (2004).

[9] Gauthier, D. J., Physics and Applications of "Slow" Light. 2nd Annual Summer School, Fitzpatrick Center for Photonics and Communication Systems, Duke University, Durham, NC, July 27, 2004

[10] G. P. Agrawal, Nonlinear fibre optics, 2nd edition (Academic Press, San Diego, CA, 1995)

[11] K. Y. Song, M. Gonzalez Herráez and L. Thévenaz, "Observation of pulse delaying and advancement in optical fibers using stimulated Brillouin scattering," Opt. Express 13, 82-88 (2005).

[12] M. Niklès, L. Thévenaz, and Ph. Robert, "Brillouin gain spectrum characterization in single-mode optical fibers," J. Lightwave Technol., LT-15, 1842-1851 (1997).

[13] M. Gonzalez Herráez, K. Y. Song and L. Thévenaz, "Optically controlled slow and fast light in optical fibers using stimulated Brillouin scattering," Appl. Phys. Lett. 87, 081113 (2005).

[14] 11. K. Y. Song, M. G. Herraez, and L. Thevenaz, "Long optically-controlled delays in optical fibers," Opt. Lett. 30, 1782-1784 (2005).

[15] M. Gonzalez Herraez, K. Y. Song, L. Thévenaz, "Arbitrary-bandwidth Brillouin slow light in optical fibers," Opt. Express 14, 1395-1400 (2006)

[16] Z. Zhu, A. M. C. Dawes, D. J. Gauthier, L. Zhang, and A. E. Willner, "Broadband SBS Slow Light in an Optical Fiber," J. Lightwave Technol. 25, 201-206 (2007)

[17] A. Zadok, A. Eyal, and M. Tur, "Extended delay of broadband signals in stimulated Brillouin scattering slow light using synthesized pump chirp," Opt. Express 14, 8498-8505 (2006)

[18] M. Gonzalez Herraez, K. Y. Song, L. Thévenaz, "Arbitrary-bandwidth Brillouin slow light in optical fibers," Opt. Express 14, 1395-1400 (2006)

[19] K. Y. Song and K. Hotate, "25 GHz bandwidth Brillouin slow light in optical fibers," Opt. Lett. 32, 217-219 (2007) 